\title{
Improving MODIS Spatial Resolution for Snow Mapping Using Wavelet Fusion and ARSIS Concept
}

\author{
Pascal Sirguey, Renaud Mathieu, Yves Arnaud, Muhammad M. Khan, \\ and Jocelyn Chanussot, Senior Member, IEEE
}

\begin{abstract}
We propose to fuse the high spatial content of two 250-m spectral bands of the Moderate Resolution Imaging Spectroradiometer (MODIS) into its five 500-m bands using wavelet-based multiresolution analysis. Our objective was to test the effectiveness of this technique to increase the accuracy of snow mapping in mountainous environments. To assess the performance of this approach, we took advantage of the simultaneity between the Advanced Spaceborne Thermal Emission and Reflection Radiometer (ASTER) and MODIS sensors. With a 15-m spatial resolution, the ASTER sensor provided reference snow maps, which were then compared to MODIS-derived snow maps. The benefit of the method was assessed through the investigation of various metrics, which showed an improvement from $3 \%$ to $20 \%$. Therefore, the enhanced snow map is of great benefit for environmental and hydrological applications in steep terrain.
\end{abstract}

Index Terms-ARSIS, Moderate Resolution Imaging Spectroradiometer (MODIS), multispectral fusion, snow, wavelet.

\section{INTRODUCTION}

$\mathbf{T}$ HE DAILY-repeat-time and multispectral capabilities of Moderate Resolution Imaging Spectroradiometer (MODIS) make it a powerful tool for the operational monitoring of snow cover properties. In order to discriminate snow from other targets, the normalized difference snow index (NDSI) is commonly used [1]-[3]. It takes advantage of the contrast between the high reflectance of snow in the green part of the visible spectrum (band 4 at $555 \mathrm{~nm}$ ) and its low reflectance in the short-wave infrared (band 6 at $1640 \mathrm{~nm}$ ) (see Fig. 1). The use of these bands constrains the mapping of snow cover at $500-\mathrm{m}$ spatial resolution. This relatively coarse spatial resolution limits our ability to accurately map snow cover, particularly when the slope becomes significant. However, the MODIS sensor also has two spectral bands at 250-m spatial resolution. In their early work, Hall et al. [4] suggested using these bands to produce more detailed maps

Manuscript received April 27, 2007; revised July 13, 2007. This work was funded by the School of Surveying, University of Otago.

P. Sirguey and R. Mathieu are with the Global Land Ice Monitoring from Space New Zealand Office, School of Surveying, University of Otago, Dunedin 9015, New Zealand (e-mail: sirpa271@ student.otago.ac.nz).

Y. Arnaud is with the Laboratoire de Glaciologie et Géophysique de l'Environnement, Institut de Recherche pour le Développement-Great Ice, 38402 Saint Martin d'Hères, France (e-mail: yves.arnaud@ird.fr).

M. Khan and J. Chanussot are with the Laboratoire Grenoblois de l'Image, de la Parole, du Signal et de l'Automatique/Centre National de la Recherche Scientifique, Département Images et Signal/Ecole Nationale Superieure d'Ingenieurs Electriciens de Grenoble, Domaine Universitaire, 38402 Saint Martin d'Hères, France (e-mail: muhammad.khan@lis.inpg.fr; jocelyn.chanussot@lis.inpg.fr).

Color versions of one or more of the figures in this paper are available online at http://ieeexplore.iee.org.

Digital Object Identifier 10.1109/LGRS.2007.908884

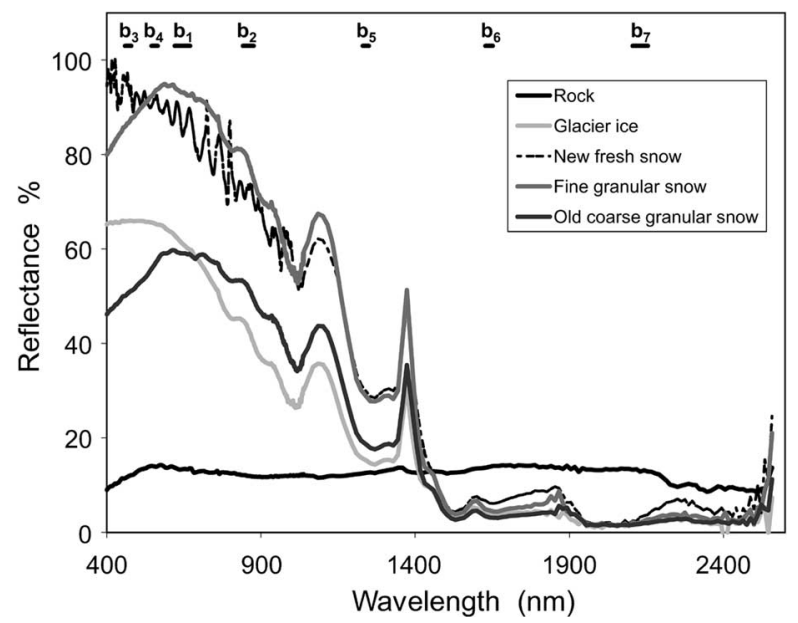

Fig. 1. Spectral signature for various snow and ice targets. Ground reflectances were measured during fieldwork on the Glenmary Glacier, South Island, New Zealand, in March 2003. The range of MODIS spectral bands 1-7 is indicated.

of snow cover. The objective of this study was to investigate whether wavelet-based image fusion between MODIS spectral bands would enable the determination of snow at $250 \mathrm{~m}$ and benefit snow cover mapping in mountainous terrain. Toward this goal, the implementation of the ARSIS concept (from its French name "Amélioration de la Résolution Spatiale par Injection de Structures"), as well as the custom-made algorithm used in this study to map snow cover, is first described. To validate the efficiency of this technique, a rigorous estimation of the improvement is carried out by comparing our MODISderived snow maps, obtained "with" and "without" the fusion process, with references obtained from simultaneous imagery acquired with the Advanced Spaceborne Thermal Emission and Reflection Radiometer (ASTER).

\section{Methodology}

\section{A. Image Fusion}

1) Principle and Methods: In Earth observations, the growing number of sensors and the variety of spatial, spectral, and temporal resolutions make satellite images suitable for fusion techniques [5]. When dealing with imagery, fusion methods usually aim to merge the rich spatial content of a high-resolution (HR) image with the rich spectral content of a low spatial resolution (LR) image (e.g., typically, the HR and the LR are the panchromatic and multispectral canals, respectively, for sensors such as Landsat, Ikonos, or Quickbird). 


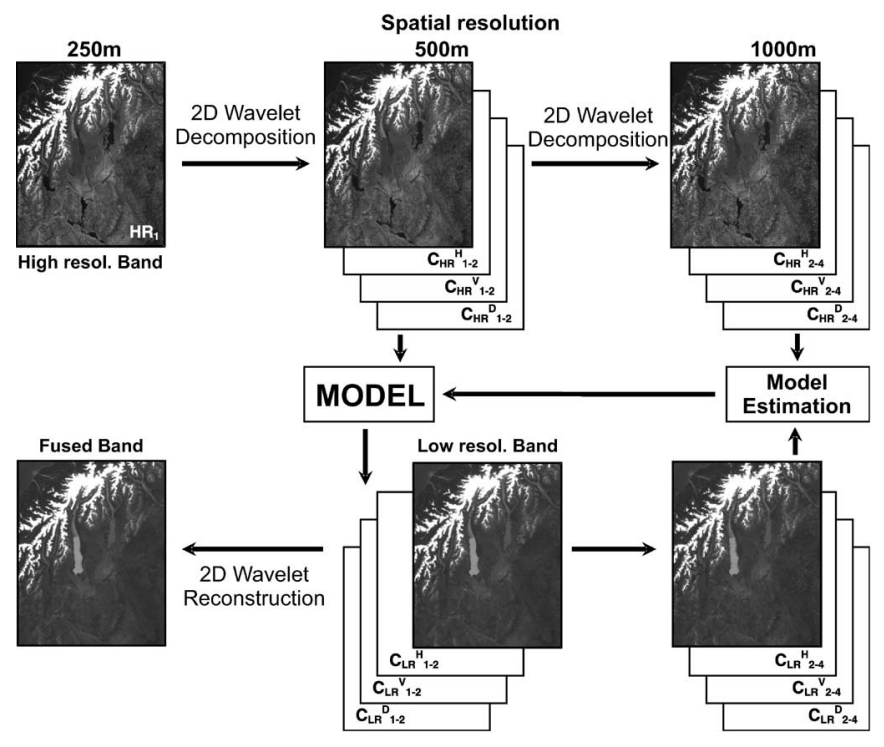

Fig. 2. ARSIS concept, as implemented in the case of MODIS using the undecimated WT (adapted from Ranchin and Wald [13]).

Several methods exist to merge multispectral (MS) bands in remote sensing. The substitution methods are based on the transformation of a three-band composite [red green blue (RGB)] into another color space, such as hue-intensitysaturation or principal component analysis. In the new space, the intensity image I or the first principal component PC1 holds the spatial information. The HR band (e.g., panchromatic) is then used as a substitute for I or PC1 before applying the inverse transformation. These methods are adequate when the HR band is highly correlated with I or PC1 [6]. Consequently, their application should be limited to sensors designed with a panchromatic canal that overlaps the whole spectral range of all the MS bands used in the three-band composite. Digressions in the spectral domain may otherwise result in significant radiometric distortions [7]. Therefore, color space substitution methods are irrelevant to MODIS, because the HR bands are not panchromatic and do not overlap spectrally with the LR bands.

To tackle this problem, multiresolution methods have been designed to extract the spatial details contained in the HR band and, subsequently, fuse or "inject" these details into the LR image. This can be achieved through different methods of analysis, such as high-pass filtering [8], the Laplacian pyramid [9], or wavelet transform (WT) [10], [11]. Each band is decomposed into a low-frequency approximation that describes the trend (radiometry) and a series of zero-mean coefficients that accounts for the high-frequency information (spatial details). Only the spatial details are incorporated into the LR image using the related reconstruction method. Merging zero-mean wavelet coefficients also avoids the bias in the radiometry of the original image, which occurs when using substitution techniques [12]. Multiresolution methods are suitable for dealing with band-to-band fusion since they extract from the image only the spatial details that exist at different scales.

2) ARSIS Concept and Implementation: Developed by Ranchin and Wald [13], the multiresolution-based concept ARSIS was implemented. As shown in Fig. 2, the decomposition of the HR image produces the first set of detail coefficients. These coefficients can be corrected by an adequate
TABLE I

SPECIFICATIONS OF THE MODIS SPECTRAL BANDS USED IN THE FUSION PROCESS AND THE MAPPING OF SNOW

\begin{tabular}{l|cc|ccccc}
\hline Band & B1 & B2 & B3 & B4 & B5 & B6 & B7 \\
\hline Center wavelength (nm) & 645 & 858 & 469 & 555 & 1240 & 1640 & 2130 \\
Spatial res. (m) & 250 & 250 & 500 & 500 & 500 & 500 & 500 \\
\hline
\end{tabular}

model before being injected in the LR image to improve the quality of the synthesized image. The modified coefficients are used along with the LR image for reconstruction, making the spatial resolution of the reconstructed image the same as that of the HR image. We used the undecimated "à trous" WT algorithm [14], combined with Daubechies D12 wavelets, as the multiresolution decomposition approach. To avoid the impact of a resampling operator, which aims to match the size of the LR and HR images, as required by the undecimated algorithm, we took advantage of the MODIS L1B Swath Product, which must be gridded onto a geographic coordinate system. All bands were first projected at a pixel size of $250 \mathrm{~m}$; the LR image used in the fusion process was provided by the approximation from a preliminary wavelet decomposition of the band to be fused (gridded at $250 \mathrm{~m}$ ). This step guarantees that the size of the images will match and that the coregistration with the detail coefficients extracted from the HR image is preserved. The identity model M1 injects unchanged coefficients and is the simplest one. However, our experimentations showed that it did not achieve good quality fusion. Thus, we implemented the more sophisticated model M2, proposed by Mangolini et al. [15], to adjust the variance and mean of the wavelet coefficients according to image-dependent factors, which are derived from a second level of decomposition as follows:

$$
\begin{aligned}
C_{\mathrm{LR}_{1-2}}^{Z} & =a^{Z} C_{\mathrm{HR}_{1-2}}^{Z}+b^{Z} \\
a^{Z} & =\sigma^{Z}(\mathrm{LR}) / \sigma^{Z}(\mathrm{HR}) \\
b^{Z} & =m^{Z}(\mathrm{LR})-a^{Z} m^{Z}(\mathrm{HR}), \quad \text { with } Z=D, V, \text { or } H
\end{aligned}
$$

where $Z$ accounts for the type of coefficient: horizontal, vertical, or diagonal. $m^{Z}(\mathrm{LR})$ and $\sigma^{Z}(\mathrm{LR})$ are the mean and standard deviation of $C_{\mathrm{LR}_{2-4}}^{Z}$, respectively. Since MODIS disposes of two HR bands and five LR bands, which are spread over the reflective part of the spectrum, we decided to use the HR band that is spectrally the closest to the LR band to be fused. This choice is subjective and disputable; no sensitivity analyses have yet been carried out to assess its relevance. Consequently, the spatial details of B1 were merged into those of B3 and B4; the spatial details of B2 were merged into those of B5, B6, and B7 (Table I).

\section{B. Postprocessing and Snow Detection}

Postprocessing refers to the steps required to actually map the snow cover. These steps are only briefly outlined. The same processing strategy was applied to both the nonfused $500-\mathrm{m}$ MODIS images and the fused 250-m MODIS images to ensure that the difference of snow mapping can only be attributed to the fusion technique.

1) Topographic and Atmospheric Correction: A rigorous 3-D topographic and atmospheric correction model has been 
TABLE II

Results for the Metrics Computed Between the ASTER REFERENCES AND MODIS SNOW MAPS DERIVED FROM NONFUSED BANDS ("WiTHOUT") AND FUSED BANDS ("WITH") FOR THE FOUR IMAGES

\begin{tabular}{|c|c|c|c|c|c|c|c|c|}
\hline Date & \multicolumn{2}{|c|}{$\overline{R^{2}}$} & \multicolumn{2}{|c|}{ MAE\% } & \multicolumn{2}{|c|}{ Q Index\% } & \multicolumn{2}{|c|}{ AUCD\% } \\
\hline$\overline{09 / 11 / 2000}$ & 0.82 & 0.91 & 8.7 & 6.5 & 90.4 & 94.8 & 38.3 & $\overline{44.2}$ \\
\hline $29 / 01 / 2002$ & 0.89 & 0.93 & 4.5 & 3.6 & 93.8 & 95.9 & 43.5 & 45.6 \\
\hline $31 / 12 / 2002$ & 0.88 & 0.93 & 6.4 & 5.0 & 94.0 & 96.1 & 43.2 & 45.2 \\
\hline $16 / 05 / 2006$ & 0.82 & 0.87 & 10.4 & 8.7 & 90.1 & 92.2 & 36.7 & 40.9 \\
\hline
\end{tabular}

adapted and implemented [16]. Richter's iterative model accounts for topographic effects, such as the illumination angle, shadows, and terrain-reflected radiations, that can be significant in mountainous areas and snowy environments. For computational purposes, we use the simple atmospheric model given by Bird and Riordan [17]. Water vapor was inferred from the MODIS image using an adaptation of the MOD05 Water Vapor Product algorithm. The Ozone column was taken from the Total Ozone Mapping Spectrometer zonal monthly average. The Aerosol optical depth was estimated from visibility observations at the nearby Mount Cook Airport and weighted according to the elevation of each pixel.

2) Linear Constrained Unmixing: The spectral unmixing technique was applied to produce maps of subpixel snow fractions, i.e., the estimate of percent snow cover within each individual pixel. We implemented a constrained linear unmixing, as described by Keshava [18]. Sea and lakes were masked. We slightly underdetermined the linear equation by selecting eight endmembers, with a specific focus on snow targets (ice, dark and bright rocks, three classes of snow describing various states of transformation, and dark and bright vegetation). The spectral signatures for each endmember were obtained from either the measured ground reflectance or the targets that were photointerpreted in corrected MODIS images. The fractions of the endmembers representing ice or snow were then summed to provide the whole snow fraction within the pixel.

\section{VALIDATION}

\section{A. Description of the Data Set}

Verifying that the procedure truly improves the determination of snow by increasing the spatial resolution is essential. Visual analysis and interpretation is a straightforward method to compare snow maps produced "with" or "without" fusion. Nevertheless, qualitative analysis is inevitably biased by the observer's experiences and subjectivity. Therefore, quantitative measures comparing the MODIS snow maps and the reference snow maps must be investigated.

Four pairs of simultaneous MODIS/ASTER acquisition that were selected at different seasons and include various conditions of snow cover were selected in our study area (see the dates in Table II). The spatial resolution of ASTER (15 m), compared with the 250 and $500 \mathrm{~m}$ of MODIS, provides a ratio of 277 and 1111 ASTER pixels, respectively, for each MODIS pixel. We therefore hypothesized that a binary classification of snow from ASTER, using a threshold of the NDSI, would provide accurate ground truth of the snow cover. The ASTER images were therefore orthorectified and converted to reflectance. The NDSI was computed using ASTER band 1 $(560 \mathrm{~nm})$ and band $4(1660 \mathrm{~nm})$. The snow was classified at a resolution of $15 \mathrm{~m}$ with a custom threshold of 0.7 . The resulting snow maps were resampled at $12.5 \mathrm{~m}$ and aggregated to 250 and $500 \mathrm{~m}$, thus providing the reference snow fraction maps of the area.

\section{B. Metrics}

1) Standard Descriptive Statistics: In our analysis, we use the mean absolute error (MAE) and the coefficient of determination $R^{2}$ as the first indicators of the performance of the fusion. The MAE is given by

$$
\text { MAE }=\frac{1}{m \times n} \sum_{i=1}^{m} \sum_{j=1}^{n}\left|x_{i j}-y_{i j}\right|
$$

where $x$ and $y$ are the snow fraction for MODIS and ASTER, respectively, and $m$ and $n$ are the number of rows and columns of the raster snow map, respectively. MAE provides an overall indication of the positive or negative errors. $R^{2}$ indicates the portion of variance in the ASTER-derived reference snow map that is accounted for in the MODIS-derived snow map.

2) Global Quality or Q Index: MODIS snow fraction maps are grayscale raster data sets. Their similarity to the ASTER reference maps can be evaluated through a global image quality index, such as the $Q$ index that was designed by Wang and Bovik [19] and generalized for the validation of multispectral image fusion by Alparone et al. [20]. This metric incorporates correlation coefficient $R$ and is sensitive to the bias and the change in contrast between the images tested. It provides a unique score that ranges between -1 and 1 , making it appropriate for ranking processing strategies according to their performances. It is defined by

$$
Q=\frac{\sigma_{x y}}{\sigma_{x} \sigma_{y}} \frac{2 \bar{x} \bar{y}}{\left[(\bar{x})^{2}+(\bar{y})^{2}\right]} \frac{2 \sigma_{x} \sigma_{y}}{\left[\sigma_{x}^{2}+\sigma_{y}^{2}\right]}
$$

where $\sigma_{x y}$ is the covariance between test image $x$ and reference image $y ; \bar{x}$ and $\bar{y}$ are the means; and $\sigma_{x}^{2}$ and $\sigma_{y}^{2}$ are the variances of $x$ and $y$, respectively.

3) Receiver Operating Characteristic (ROC) Curves: Another valuable tool to compare classifiers according to their performance is ROC curves, as they provide a comprehensive interpretation of confusion matrices [21]. When two classes are defined, the hit rate and false alarm rate can be computed. These represent the probability of the classifier to categorize a pixel within the right or the wrong class. These probabilities define a single point in the ROC space. If the classifier also provides the probability of belonging to one or the other class, then a continuous ROC curve can be drawn by increasing the threshold of this probability.

In our case, such an approach cannot be applied directly since our classifier is continuous. Indeed, the snow fraction within a pixel can take any value in the range $[0,1]$. To tackle this issue, the reference snow maps were equally segmented into 11 classes (ten thresholds: $\{0.5,0.15, \ldots, 0.95\}$ ). To accommodate the nonuniform pixel distribution in each class, equal numbers of pixels were randomly selected in each class, based on the smallest class. For each threshold, the classes 
(a)

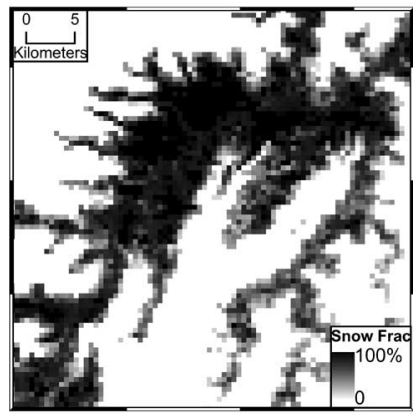

(c)

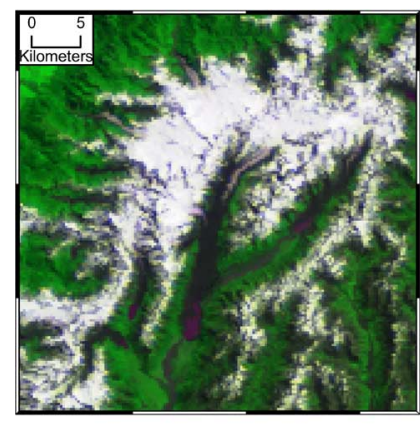

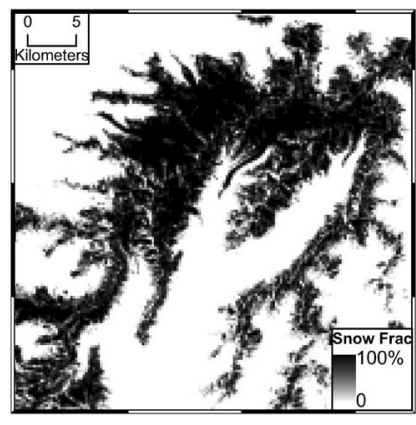

(b)

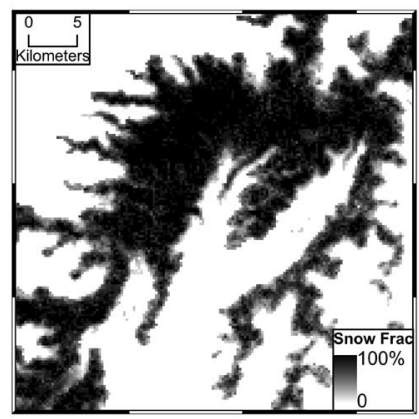

(d)
Fig. 3. Visual performance in estimating the snow fraction (Mount Cook Area, South Island, New Zealand; December 31, 2002 22:35 GMT). (a) False color RGB composite from MODIS bands 1, $2(250 \mathrm{~m}$ ), and 3 (fused to $250 \mathrm{~m}$ ). (b) Reference from ASTER $(15 \mathrm{~m})$ aggregated at $250 \mathrm{~m}$. (c) MODIS-derived snow map without fused bands at $500 \mathrm{~m}$. (d) MODIS-derived snow map with fused bands at $250 \mathrm{~m}$.

are gathered into two sets of pixels that are defined as being lower or greater than the given threshold. A ROC curve can then be drawn from the histograms of the MODIS-derived snow fraction for each of these two sets.

The visual interpretation of the curves for both products, "with" or "without" fusion, enables us to identify the process that performs best. This 2-D representation can also be reduced to a single scalar value by computing the Area Under the Curve and the no-Discrimination (or "pure guessing") line (AUCD). This metric is a statistical indicator of the ability of a classifier to correctly sort pixels into their respective classes.

\section{RESUlT AND DiscusSION}

For one MODIS image, as shown in Fig. 3(a), and the corresponding ASTER reference snow map [Fig. 3(b)], the MODIS-derived snow maps at $500 \mathrm{~m}$ [Fig. 3(c)] and $250 \mathrm{~m}$ [Fig. 3(d)] illustrate the resolution improvement obtained with the fusion. The visual interpretation of the snow maps for each granule leads us to consider the fused product as "significantly better" than the nonfused one, because more details could be seen in the 250-m maps. This richer spatial information generally matched well the features that are depicted in the 15-m ASTER reference image. From a quantitative point of view, however, comparing images of different spatial resolutions raises issues that are related to multiscale analysis. Not all metrics are suitable in assessing the gain of information generated by an increase in spatial resolution. For instance, if we imagine having only one pixel for the whole area to which we apply

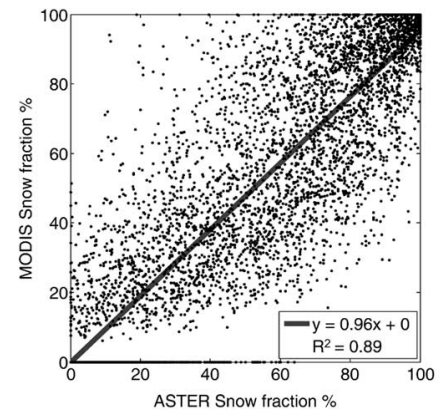

(a)

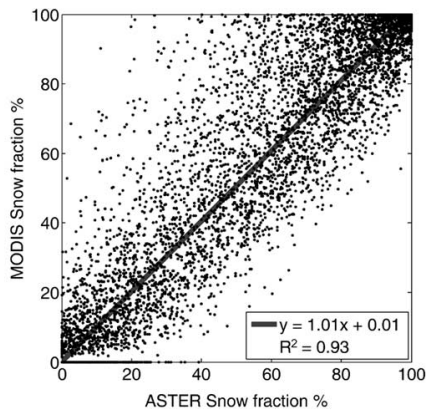

(b)
Fig. 4. Linear regression. (a) Snow fraction from MODIS 500-m bands versus the ASTER reference and (b) snow fraction from MODIS 250-m fused bands aggregated at $500 \mathrm{~m}$ versus the ASTER reference.

the unmixing method, the fraction of snow is likely to be close to the true value if the linear model is robust. In this case, the performance, in terms of the estimation of the snow cover area (SCA), would be high. However, the quantity of information in terms of spatial distribution would be dramatically reduced. On the other hand, when applying unmixing to smaller but more abundant pixels, the uncertainties originating from each pixel would be summed, and a slight bias in the unmixing process would be multiplied as the spatial resolution increases. This may result in a possible larger digression of the SCA; however, the determination of snow presence on a pixel basis will be dramatically improved. In our investigation, two images (11/09/00 and 29/01/02) showed a $1.5 \%$ and $1 \%$ improvement in the estimation of their SCA. However, there was no, or very small, deterioration observed for the two other images. Although the SCA, overall, dropped from $4.1 \%$ overestimation without fusion to $1.9 \%$ with fusion, integral metrics such as the SCA are not appropriate for assessing the improvement of snow cover determination gained by the fusion process. Therefore, we used metrics that work at the "pixel scale" and account for pixel-to-pixel digression (e.g., $R$, MAE, and $Q$ ), because they are more sensitive to the performance in terms of spatial distribution of the snow fraction and thus are more appropriate for assessing the accuracy of the mapping process. Nevertheless, in order to be compared, all metrics should be established based on maps having the same number of samples. The 250-m snow maps obtained for MODIS fused bands were therefore aggregated to $500 \mathrm{~m}$ prior to computing the metrics.

The plots of the snow fractions from the MODIS-derived snow maps versus the true equivalent ASTER snow fractions are given in Fig. 4. Fig. 4(a) represents the 500-m map obtained from the nonfused bands, and Fig. 4(b) shows the 250-m map obtained from the fused bands. The product obtained with the fusion method exhibits less point dispersion around the $1: 1$ ratio line, meaning a better determination. The results for the various metrics and the four granules investigated are given in Table II. For all granules, we observe a favorable evolution of coefficient $R^{2}$ when the fusion was processed. An average increase of $6.1 \%$ was obtained. Likewise, the MAE improved significantly, dropping by about $20 \%$ on average. The evolution of these two standard statistics illustrates the reduced dispersion shown in the estimation of the snow fraction (Fig. 4). The $Q$ index also increased by $3 \%$. For all investigated thresholds (examples of the ROC curves for two thresholds are given in Fig. 5), 


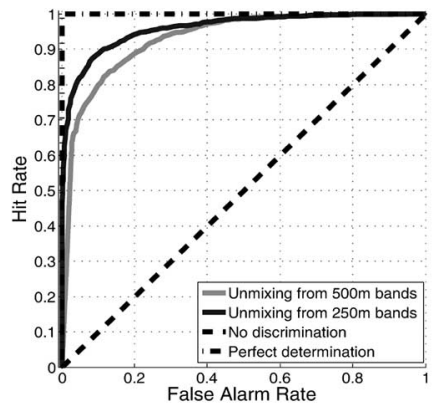

(a)

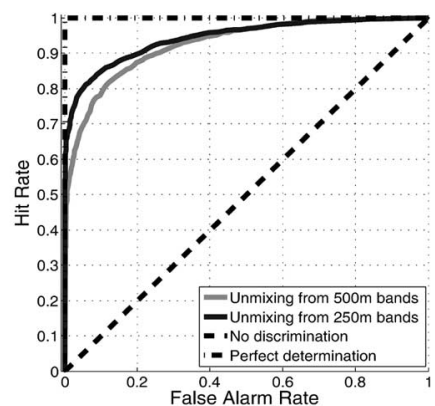

(b)
Fig. 5. ROC curves for two thresholds at 0.45 and 0.65 (Mount Cook Area, South Island, New Zealand; December 31, 2002).

the ROC curves of the fused bands also tended toward a better determination of the snow classes. The scores reported in Table II for AUCD are the average values of all ROC curves, increasing by $9 \%$ on average. The differences observed in the magnitude of change for each metric illustrate the difficulty of finding a perfect indicator to assess the effectiveness of this approach. Nevertheless, using various metrics that show a coherent trend confirms the robustness of the method and objectively validates a significant added value. However, although all metrics show that a better estimation of the snow fraction within the pixel can be achieved at the 500-m spatial resolution, by aggregating the $250-\mathrm{m}$ spatial-resolution products to $500 \mathrm{~m}$, none of them truly accounts for the fact that the snow fractions can now be mapped at $250 \mathrm{~m}$. At $250 \mathrm{~m}$, the $R^{2}$ between the MODIS-derived snow maps "with" fusion and the ASTER reference reached 0.84 , on average, proving that the method is still competitive at this scale. In the future, however, a metric that would score the snow maps without the need to be computed at the same spatial resolution would be interesting. An average distance between the snowlines extracted from the MODIS-derived snow map and the 15-m ASTER snow map could be investigated as a possible relevant metric to assess the performance of snow cover definition. On the other hand, the multiplication of the processing step makes this algorithm time consuming. The unmixing technique also has constraints with regard to the number of endmembers, which limit its use to relatively small areas where a limited number of land cover types are known. This method is therefore appropriate, at this time, only for use at the local scale.

\section{CONCLUSION}

In this letter, we investigated the improvement that can be achieved by using wavelet fusion between MODIS spectral bands for mapping snow cover at a higher spatial resolution. The ARSIS fusion concept, along with the M2 model, proved to be efficient. Qualitative analyses and rigorous metrics have been used to assess this result. The use of simultaneous ASTER acquisition also enabled us to compare our products with detailed reference maps without time lag. All validation techniques showed a significant trend toward improvement and demonstrate that the snow fraction can be more accurately estimated with the fused 250-m MODIS-derived snow map. The enhanced snow map is therefore of great benefit for environmental and hydrological applications in steep terrain.

\section{ACKNOWLEDGMENT}

The authors would like to thank Prof. M. Fily, Head of the Laboratoire de Glaciologie et Géophysique de l'Environnement (Grenoble, France), for his support.

\section{REFERENCES}

[1] R. G. Crane and M. R. Anderson, "Satellite discrimination of snow/cloud surfaces,” Int. J. Remote Sens., vol. 5, no. 1, pp. 213-223, 1984.

[2] J. Dozier, "Spectral signature of alpine snow cover from the Landsat thematic mapper," Remote Sens. Environ., vol. 28, pp. 9-22, 1989.

[3] D. K. Hall, G. Riggs, V. V. Salomonson, N. E. DiGirolamo, and K. J. Bayr, "MODIS snow-cover products," Remote Sens. Environ., vol. 83, no. 1, pp. 181-194, Nov. 2002.

[4] D. K. Hall, G. A. Riggs, and V. V. Salomonson, "Development of methods for mapping global snow cover using moderate resolution imaging spectroradiometer data," Remote Sens. Environ., vol. 54, no. 2, pp. 127-140, Nov. 1995.

[5] L. Wald, Data Fusion: Definitions and Architectures (Fusion of Images of Different Spatial Resolutions). Paris, France: Les Presses de l'Ecole des Mines de Paris, 2002.

[6] M. González-Audicana, J. L. Saleta, R. G. Catalán, and R. García, "Fusion of multispectral and panchromatic images using improved IHS and PCA mergers based on wavelet decomposition," IEEE Trans. Geosci. Remote Sens., vol. 42, no. 6, pp. 1291-1299, Jun. 2004.

[7] T.-M. Tu, P. S. Huang, C.-L. Hung, and C.-P. Chang, "A fast intensityhue-saturation fusion technique with spectral adjustment for IKONOS imagery," IEEE Geosci. Remote Sens. Lett., vol. 1, no. 4, pp. 309-312, Oct. 2004.

[8] P. S. J. Chavez, S. C. Sides, and J. A. Anderson, "Comparison of three different methods to merge multiresolution and multispectral data: Landsat TM and SPOT panchromatic," Photogramm. Eng. Remote Sens., vol. 57, no. 3, pp. 265-303, 1991.

[9] B. Aiazzi, L. Alparone, F. Argenti, and S. Baronti, "Wavelet and pyramid techniques for multisensor data fusion: A performance comparison varying with scale ratios," in Proc. SPIE_Image Signal Process. For Remote Sensing V, S. B. Serpico, Ed., Dec. 1999, vol. 3871, pp. 251-262.

[10] T. Ranchin and L. Wald, "The wavelet transform for the analysis of remotely sensed images," Int. J. Remote Sens., vol. 14, no. 3, pp. 615619, Feb. 1993.

[11] B. Garguet-Duport, J. Girel, J.-M. Chassery, and G. Pautou, "The use of multiresolution analysis and wavelet transform for merging SPOT panchromatic and multispectral imagery data," Photogramm. Eng. Remote Sens., vol. 62, no. 9, pp. 1057-1066, 1996.

[12] S. Li, J. T. Kwok, and Y. Wang, "Using the discrete wavelet frame transform to merge Landsat TM and SPOT panchromatic images," Inf. Fusion, vol. 3, no. 1, pp. 17-23, Mar. 2002.

[13] T. Ranchin and L. Wald, "Fusion of high spatial and spectral resolution images: The ARSIS concept and its implementation," Photogramm. Eng. Remote Sens., vol. 66, no. 1, pp. 49-61, 2000.

[14] M. J. Shensa, "The discrete wavelet transform: Wedding the a trous and Mallat algorithms," IEEE Trans. Signal Process., vol. 40, no. 10, pp. 2464-2482, Oct. 1992.

[15] M. Mangolini, T. Ranchin, and L. Wald, "Procédé et dispositif pour l'amélioration de la résolution spatiale d'images à partir d'autres images de meilleure résolution spatiale," French Patent 92-13961, Nov. 1992.

[16] R. Richter, "Correction of satellite imagery over mountainous terrain," Appl. Opt., vol. 37, no. 18, pp. 4004-4015, Jun. 1998.

[17] R. E. Bird and C. Riordan, "Simple solar model for direct and diffuse irradiance on horizontal and tilted planes at the earth's surface for cloudless atmosphere," J. Clim. Appl. Meteorol., vol. 25, pp. 87-97, 1986.

[18] N. Keshava, "A survey of spectral unmixing algorithms," Lincoln Lab. J., vol. 14, no. 1, pp. 55-78, 2003.

[19] Z. Wang and A. C. Bovik, "A universal image quality index," IEEE Signal Process. Lett., vol. 9, no. 3, pp. 81-84, Mar. 2002.

[20] L. Alparone, S. Baronti, A. Garzelli, and F. Nencini, "A global quality measurement of pan-sharpened multispectral imagery," IEEE Geosci. Remote Sens. Lett., vol. 1, no. 4, pp. 313-317, Oct. 2004.

[21] T. Fawcett, "ROC Graphs: Notes and Practical Considerations for Researchers," HP Lab., Palo Alto, CA, Tech. Rep., 2004. 